\title{
4-D STEM Analyses of Cylindrical Specimens for Atom Probe Tomography
}

Brian Gorman ${ }^{1}$, Megan Holtz ${ }^{1}$, Christopher J. K. Richardson ${ }^{2}$, Chomani Gaspe ${ }^{3}$, Edwin Supple ${ }^{1}$ and Robert Butera ${ }^{3}$

${ }^{1}$ Colorado School of Mines, United States, ${ }^{2}$ Laboratory for Physical Sciences, United States,

${ }^{3}$ Laboratory for Physical Science, United States

Atom Probe Tomography (APT) data reconstruction using geometrical models of field evaporation has many limitations due to the number of free empirical values. Correlating information from Transmission Electron Microscopy (TEM) and APT on the same specimen has shown the ability to determine the specimen volume using pre- and post-APT imaging, thus limiting the free variables. Additionally, a recent method using TEM and electron diffraction data has made it possible to incorporate atomic positions within the APT data reconstruction volumes [1], a method dubbed STEM-centric atom placement [2].

In this work, we expand upon the idea of STEM-centric atom placement by utilizing 4-D STEM data, where convergent beam electron diffraction data is collected at sub-nm spacings across the cylindrical atom probe specimen before APT analysis is conducted. In this work, a SiGe metamorphic buffer layer on a Si (100) substrate was grown using molecular beam epitaxy and prepared for STEM and APT using standard focused ion beam (FIB) methods. The sample was prepared on Au TEM grids and followed by $5 \mathrm{kV}$ cleaning steps. 4-D STEM analyses were conducted using a Thermo Fisher Scientific Talos F200X STEM at $200 \mathrm{keV}$ and a Ceta camera at 512 x 512 pixel density. Bright field, dark field, and differential phase contrast micrographs were produced from the data using LiberTEM [3]. Strain analyses were conducted using Exit Wave Phase Cepstrum (EWPC) methods [4]. APT data was collected on a Cameca LEAP $4000 \mathrm{XSi}$ using $25 \mathrm{pJ}$ of laser energy per pulse and a base temperature of $40 \mathrm{~K}$.

Figure 1 illustrates the ability of 4-D STEM to identify the atomic structure within the cylindrical specimens. Crystallographic phase and orientations can be determined directly from the diffraction patterns, similar to those produced using Transmission Kikuchi Diffraction, although at improved spatial resolution better than $0.25 \mathrm{~nm}$. Bright field and dark field micrographs can be produced from each phase or orientation by mapping the intensity of individual diffraction peaks. Artifacts from FIB specimen preparation can be identified by amorphous diffraction patterns (Figure 2), also illustrating the degree of specimen damage that occurs during low-kV FIB cleanup steps.

Local fields can also be mapped using 4-D STEM analyses, especially at internal interfaces and at the solid-vacuum interfaces. Future 4-D STEM experiments analyzing specimens while under bias may lead to greater understanding of APT field evaporation processes. Importantly, the crystallographic orientations relative to the specimen evaporation axes gives information about the correct atomic spacings and densities during the reconstruction processes. Using EWPC, we show in Figure 2 that the lattice parameter can be mapped to better than 0.1 Angstroms throughout the entire specimen, thus giving valuable information about atoms locations prior to APT analyses. Combining this information along with post-APT STEM imaging confines the APT analysis volume and atom locations prior to isotopic APT data incorporation, taking an important step towards accurate Atomic Scale Tomography. [5] 

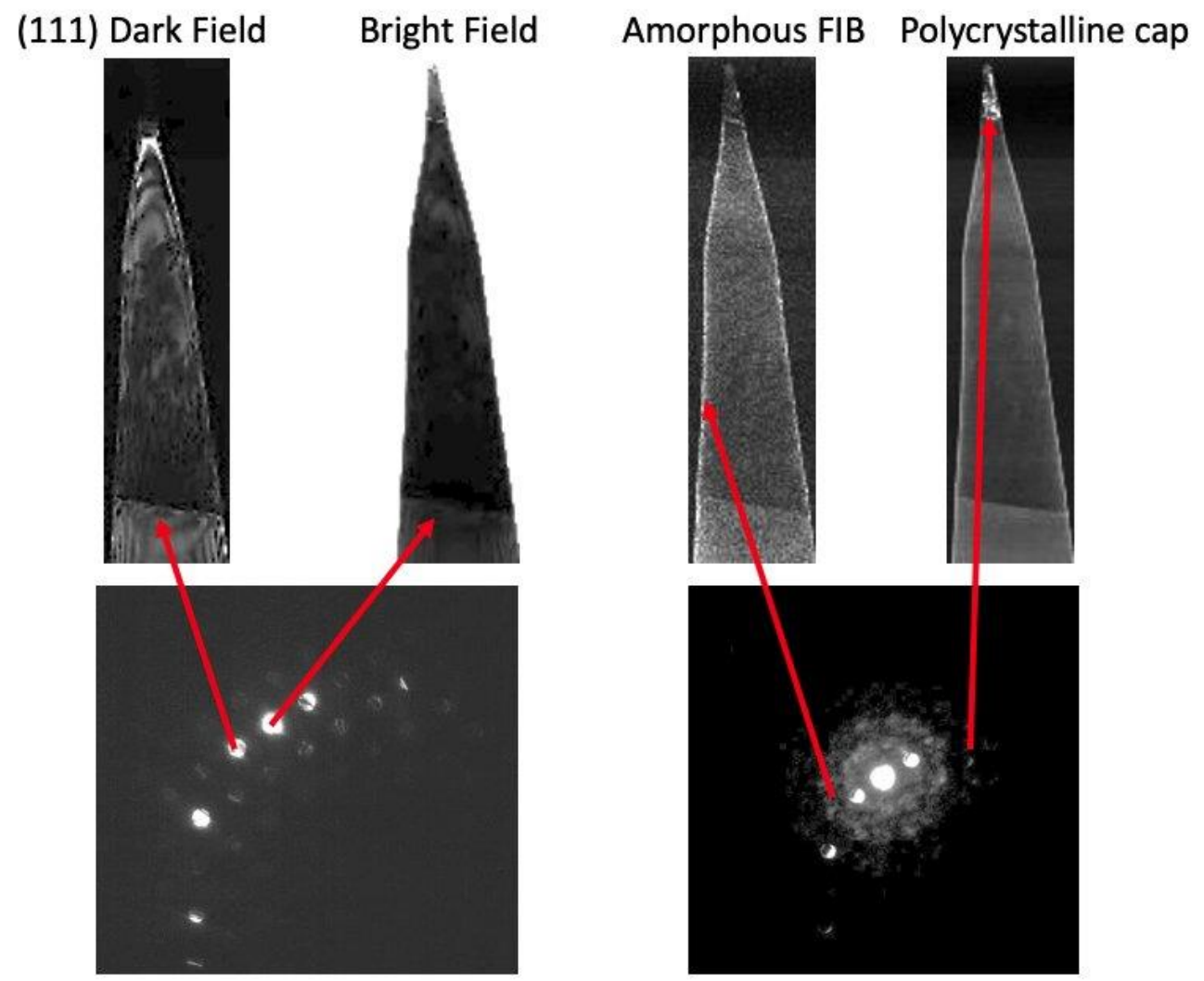

Figure 1. Figure 1. 4-D STEM data analysis produces Bright Field and Dark Field micrographs of an epitaxial SiGe buffer layer grown on a Si (100) substrate. Principal component analysis of the EWPC data illustrates FIB amorphization on the outside edges of the specimen and the polycrystalline metal capping layer used to protect the surface during specimen preparation. Horizontal field width $=100 \mathrm{~nm}$.

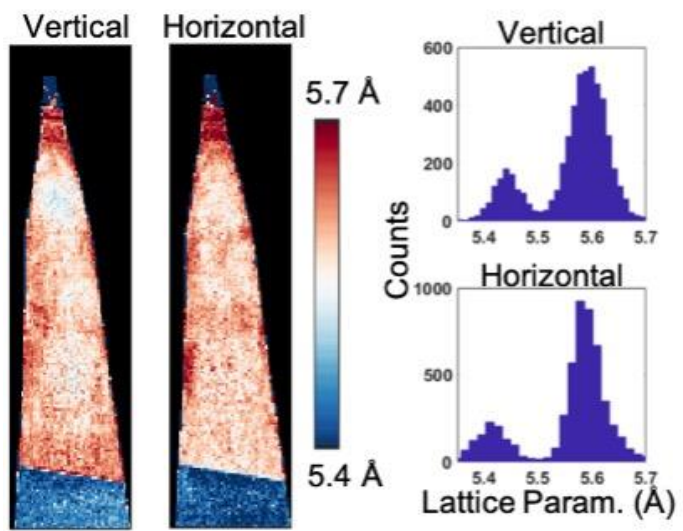

Figure 2. Figure 2. EWPC mapping and histograms of the lattice parameters in the out-of-plane (vertical) and inplane (horizontal) directions show a relaxed SiGe film with a strain gradient near the tip of the specimen.

\section{References}

[1] Ceguerra, A., Breen, A., Cairney, J., Ringer, S. \& Gorman, B. (2021). Integrative Atom Probe Tomography using STEM-Centric Atom Placement as a Step Towards Atomic-Scale Tomography. MICROSCOPY AND MICROANALYSIS 27, 140-148. 
[2] T. F. Kelly, Gorman, B. P. \& Ringer, S. P. (2021). Atomic Scale Analytical Tomography. Cambridge University Press.

[3] Clausen, A., Weber, D., Ruzaeva, K., Migunov, V., Baburajan, A., Bahuleyan, A., Caron, J., Chandra, R., Dey, S., Halder, S., Levin, B. D. A., Nord, M., Ophus, C., Peter, S., Schyndel van, J., Shin, J., Sunku, S., Müller-Caspary, K. \& Dunin-Borkowski, R. E. (2020). LiberTEM/LiberTEM: 0.5.1. Zenodo https://zenodo.org/record/3982290\#.X4364EJKhTY

[4] Padgett, E., Holtz, M. E., Cueva, P., Shao, Y.-T., Langenberg, E., Schlom, D. G. \& Muller, D. A. (2020). The exit-wave power-cepstrum transform for scanning nanobeam electron diffraction: robust strain mapping at subnanometer resolution and subpicometer precision. Ultramicroscopy 214, 112994. [5] This work was funded by the Laboratory for Physical Science under contract \#H98230-19-C-0428 